\title{
Brookhaven National Laboratory (BNL) Proton Beam Characteristics and Dynamics: A First Look
}

\author{
by \\ Kevin R. Vixie and Gary Sandine \\ Los Alamos National Laboratory
}

\begin{abstract}
This report is the first in a series addressing issues that affect the quantitative conclusions of proton radiographic analysis. In this paper we begin studying the dynamics and measurement of the proton beam profile. We conclude with a plan for further study.
\end{abstract}




\section{INTRODUCTION}

\section{Introduction}

This report is the first in a series addressing issues that arise in the analysis of data collected in the proton radiography experiments. The ultimate goal of these experiments is to determine object density and material identification (ID) as a function of location. Part of this goal, necessary for the analysis to be useful, is the determination of error bars for the reconstructed densities. Integral to this is a sensitivity analysis of the whole experiment and analysis method that will rigorously tell us where improvements need to be made and what practical limits there are to the usefulness of the data.

Remark on terminology: Throughout this report, we will refer to the output of the reconstruction analysis and algorithms as "final numbers" and the error bounds as "error bars". By error bound I mean an upper bound on the maximum difference between the true quantity and the predicted quantity using some specified norm. BNL is Brookhaven National Laboratory.

In this report we address the issue of beam variability and measurement uncertainties. More specifically, we take a preliminary look at the variation of the impingent proton beam and the size of the measurement errors. The beam is recorded at several locations. (See Fig. 1 below.) The first such record is

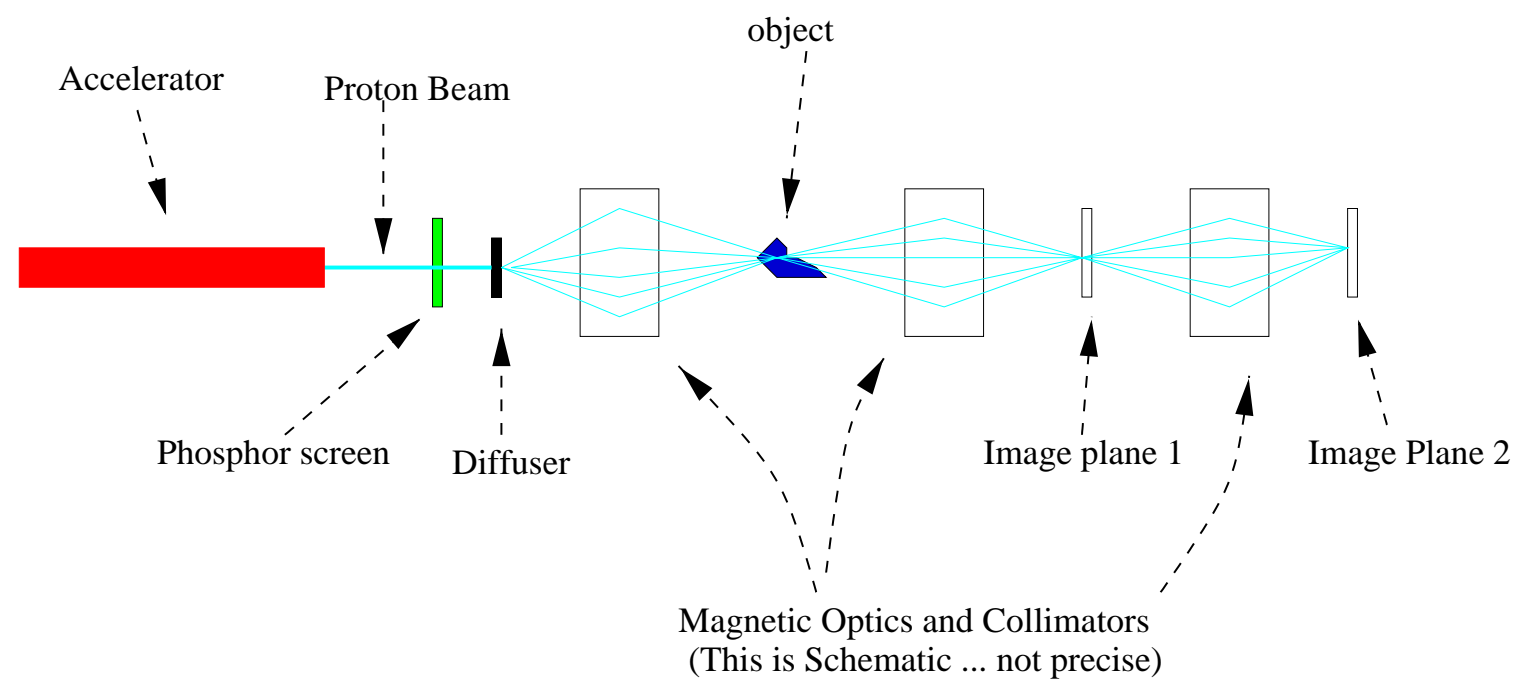

Fig. 1: Schematic of the beam, object, and image planes 


\section{INTRODUCTION}

obtained as the beam passes through a phosphor screen in front of the diffuser plate. A later report will address this beam profile and its relation to the beam profile that hits the experimental object. Next the beam hits the diffuser and spreads to an approximately Gaussian profile before heading into the magnetic lenses which eventually deliver it to the object plane. After being attenuated by the object, further lenses deliver the image to an image plane in which 4 plates are located. These 4 plates record the same image (modulo variations in plate sensitivities). The beam then is delivered by magnetic lenses to another image plane where 4 additional plates again record the attenuated beam. There are also collimators along the way that permit material ID to be done with the images from image planes 1 and 2 .

To obtain beam profiles which are subsequently used in the radiographic reconstruction process, radiographs are made with no object in the object plane. The resulting radiograph of the post-diffuser beam profile is used to adjust for the nonuniformity of the beam profile in radiographs where objects are in place. If the beam profile is not a function of time and there are no measurement errors, this adjustment works perfectly. In reality, neither of these conditions are met.

The phosphor recording plates are made by Fuji for medical radiography. The phosphor in the plate is moved to an excited state by ionizing radiation. Exposure to light prompts the decay through the release of red light to the non-excited state. In this way, excitation can be measured by collecting the red light that is stimulated by a laser beam scanned across the plate. Exposure to bright light for a few minutes completely "erases" the excitation, whereupon the plate can be used again.

The plates themselves appear to be an important source of variability. In fact, previous studies [1] seem to indicate that the plates vary in sensitivity across their extent by as much as $10 \%$. There is also a mysterious variation in plate response from experiment to experiment that has been identified but not explained (to date). The plates have a slow and fast time governing the decay of excitation. Some of these issues have already been studied by Alexander Saunders [1]. All these issues will be examined more closely in coming reports. What can be stated unequivocally though is that the final word on beam variability can only achieved when the plates are completely characterized. In 


\section{DESCRIPTION OF DATA}

this report we examine what can be understood of the beam variability without first settling these plate issues.

\section{Description of data}

The Brookhaven experiments included eleven beam shots. Of these, nine were good, meaning that some of the plates recorded the event. There was one record of the beam without the diffuser in place. Of the remaining eight shots with the diffuser in place, five had less than the full number of eight plates recording the event. The good data were composed of forty plates, representing the eight beam shots over a span of five days; we give the dates and times of the shots in Table 1 (or as a graph for visually oriented readers in Fig. 2). The diffuser for all these experiments was a half-inch thick sheet of Tantalum.

\begin{tabular}{|l|lll|}
\hline run & when & \\
\hline \hline 6986 & $8 / 19$ & $10: 51$ & hrs \\
\hline 7000 & $8 / 20$ & $00: 10$ & hrs \\
\hline 7050 & $8 / 20$ & $20: 45$ & hrs \\
\hline 7061 & $8 / 20$ & $23: 00$ & hrs \\
\hline 7066 & $8 / 21$ & $02: 50$ & hrs \\
\hline 7072 & $8 / 21$ & $06: 00$ & hrs \\
\hline 7130 & $8 / 22$ & $05: 50$ & hrs \\
\hline 7209 & $8 / 23$ & $05: 00$ & hrs \\
\hline
\end{tabular}

Table 1: Time information

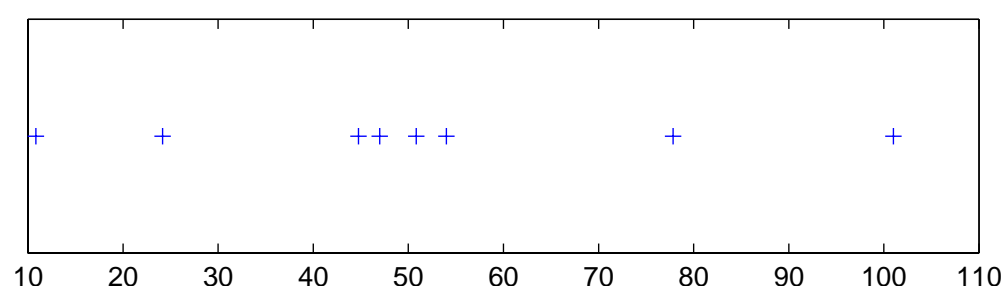

Fig. 2: Time line showing times of beam shots in hours from 00:00 of 8/19/1999 


\section{ANALYSIS AND RESULTS}

After exposure to the beam, the plates were removed from the image planes and placed two at a time on a drum scanner. The plates were then scanned, generating several TIFF files, each looking like the image (minus the black squares) in Fig. 3 below. From each raw data file, we extracted the left and

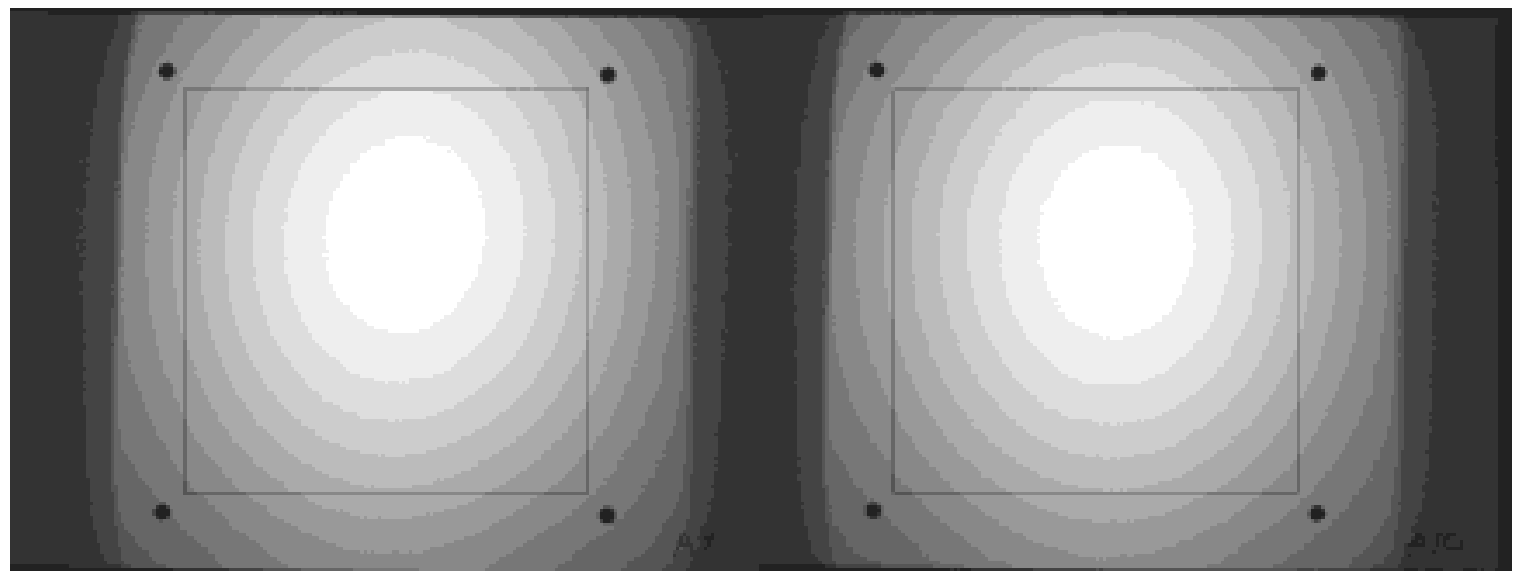

Fig. 3: (Run 6986) Portion of data used for fit

right images, and from those we extracted square sections represented by the black squares in Fig. 3. The resulting chunks of raw data were $540 \times 540$ pixels. The size and location of those chunks were chosen so as to exclude the dark registration circles, to include the centers of the beam profiles, and to do so without change from TIFF file to TIFF file (i.e. without changing coordinates for the box). It is important to note that the TIFF files were generated by first hand-placing the plates on a drum which then was automatically scanned, the results of which were the TIFF files. This process led to visible variation in the position and rotation of the plates insofar as their TIFF file coordinates are concerned. The log books can be obtained at [2] and the data can be found at $[3]$.

\section{Analysis and Results}

As a first step, we simply fit 7-parameter, 2-d Gaussians to the measured profiles. To do this, we used IDL (Interactive Data Language, by Research 


\section{ANALYSIS AND RESULTS}

Systems, Inc.) and its gauss2dfit function. The function fits a model of the form

$$
f(x, y)=s+h \exp \left(-\frac{1}{2}\left(\left(x^{\prime} / \sigma_{x}\right)^{2}+\left(y^{\prime} / \sigma_{y}\right)^{2}\right)\right),
$$

where

$$
\begin{aligned}
& x^{\prime}=\left(x-\mu_{x}\right) \cos \theta-\left(y-\mu_{y}\right) \sin \theta \\
& y^{\prime}=\left(x-\mu_{x}\right) \sin \theta+\left(y-\mu_{y}\right) \cos \theta .
\end{aligned}
$$

The parameter $s$ is a vertical shift, $h$ is the height of the Gaussian, $\sigma_{x}$ and $\sigma_{y}$ represent the spread in the $x$ and $y$ directions, $\left(\mu_{x}, \mu_{y}\right)$ represents the center, and $\theta$ allows for a rotation.

The results from the beam shot analysis are contained in Table 2, which appears on the next page. We now list specific, significant observations.

- Variation in plate responses. The data from plates A21 and A22 from runs 7061 (8/20 23:00), 7066 (8/21 02:50), and 7072 (8/21 06:00) shows that the plates themselves affect the parameter values. In run 7066, the $\sigma_{x}$ and $\sigma_{y}$ values from plate A21 are $1 \%$ and $0.3 \%$ lower, respectively, than those from plate A22, whereas in run 7072 , they are $2 \%$ and $0.7 \%$ lower. However, in run 7061, the $\sigma$-values from data on plate A22 were lower than those from plate A21 $\left(\sigma_{x}\right.$ was $2 \%$ lower, and $\sigma_{y}$ was $0.2 \%$ lower). Another example of response variation can be seen in the results from run 7061. The $\sigma_{x}$ values corresponding to run 7061, image location 1 in Table 2 (page 7 ) are reasonably consistent, except the value computed from the measurement on plate A25 is over 5.5 pixels larger than the others. This difference is observed in spite of the fact that these plates were stacked together at image location 1.

- Warping. In Fig. 4 (on page 8), we show the data from plates A26 and A25 corresponding to run 7061. The four black "holes" which mark the position of the registration posts in the data were in a square pattern of approximately 590 pixels per side. Close examination of the plates with superimposed squares shows that the registration marks are not in a square pattern. This result probably stems from errors in the hand placement of the plate in the scanning process. (The four holes in left side of Fig. 4 are very close to square, but the holes in the right side are not some are as many as five pixels from where they "should" be). 


\section{ANALYSIS AND RESULTS}

\begin{tabular}{|c|c|c|c|c|c|c|c|c|c|}
\hline run & il & plate & $s$ & $h$ & $\mu_{x} \quad(\mathrm{p})$ & $\mu_{y}(\mathrm{p})$ & $\sigma_{x}(\mathrm{p})$ & $\sigma_{y}(\mathrm{p})$ & $\theta$ (rad) \\
\hline \multirow[t]{2}{*}{ 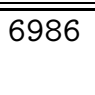 } & \multirow[t]{2}{*}{ il2 } & A9 & $1.654 \mathrm{e}+05$ & $1.377 e+08$ & 293.213 & 343.396 & 160.904 & 199.616 & 0.117 \\
\hline & & A10 & $2.260 \mathrm{e}+06$ & $1.358 \mathrm{e}+08$ & 295.806 & 335.227 & 156.040 & 193.040 & 0.059 \\
\hline \multirow[t]{2}{*}{7000} & \multirow[t]{2}{*}{ il1 } & A4 & $7.791 \mathrm{e}+06$ & $8.727 e+07$ & 282.690 & 78.959 & 153.406 & 174.728 & 0.081 \\
\hline & & A3 & $7.624 \mathrm{e}+06$ & $9.032 \mathrm{e}+07$ & 282.057 & 80.662 & 155.166 & 174.664 & 0.056 \\
\hline \multirow[t]{4}{*}{7050} & \multirow{2}{*}{ il1 } & A7 & $5.154 \mathrm{e}+06$ & $2.585 e+08$ & 244.090 & 248.979 & 154.063 & 191.132 & 0.017 \\
\hline & & A8 & $5.419 \mathrm{e}+06$ & $2.497 e+08$ & 247.479 & 251.732 & 152.639 & 189.797 & -0.003 \\
\hline & \multirow[t]{2}{*}{ il2 } & A18 & $3.705 e+06$ & $2.505 \mathrm{e}+08$ & 283.588 & 292.148 & 157.703 & 190.497 & 0.031 \\
\hline & & A17 & $5.154 \mathrm{e}+06$ & $2.555 \mathrm{e}+08$ & 289.156 & 292.127 & 154.663 & 188.757 & -0.011 \\
\hline \multirow[t]{8}{*}{7061} & \multirow[t]{4}{*}{ il1 } & $\overline{A 26}$ & $4.242 \mathrm{e}+06$ & $3.888 \mathrm{e}+08$ & 258.619 & 250.715 & 156.771 & 183.441 & 0.018 \\
\hline & & A25 & $2.318 \mathrm{e}+06$ & $4.026 \mathrm{e}+08$ & 268.837 & 256.619 & 162.319 & 183.262 & 0.072 \\
\hline & & A23 & $6.181 \mathrm{e}+06$ & $4.123 e+08$ & 260.917 & 254.392 & 156.646 & 181.396 & 0.018 \\
\hline & & A24 & $6.874 \mathrm{e}+06$ & $4.100 e+08$ & 255.307 & 251.184 & 156.206 & 181.055 & -0.009 \\
\hline & \multirow[t]{4}{*}{ i12 } & A1 & $4.504 \mathrm{e}+06$ & $3.678 e+08$ & 280.815 & 293.170 & 158.505 & 181.034 & 0.029 \\
\hline & & $\overline{\mathrm{A} 2}$ & $7.061 \mathrm{e}+06$ & $4.025 \mathrm{e}+08$ & 286.462 & 299.012 & 157.593 & 181.638 & 0.143 \\
\hline & & A22 & $6.520 \mathrm{e}+06$ & $4.126 \mathrm{e}+08$ & 277.470 & 292.172 & 157.764 & 179.844 & 0.025 \\
\hline & & A21 & $6.106 \mathrm{e}+06$ & $4.267 e+08$ & 284.114 & 282.636 & 160.987 & 180.139 & -0.050 \\
\hline \multirow[t]{8}{*}{7066} & \multirow[t]{4}{*}{ il1 } & $\overline{\mathrm{A} 21}$ & $1.158 \mathrm{e}+07$ & $6.406 \mathrm{e}+08$ & 285.243 & 239.980 & 155.414 & 185.424 & 0.006 \\
\hline & & A22 & $1.098 \mathrm{e}+07$ & $6.806 e+08$ & 282.580 & 238.941 & 157.059 & 186.093 & 0.006 \\
\hline & & A26 & $1.503 e+07$ & $6.816 e+08$ & 284.644 & 240.219 & 156.490 & 186.976 & 0.029 \\
\hline & & A25 & $1.418 \mathrm{e}+07$ & $6.928 \mathrm{e}+08$ & 289.110 & 238.502 & 155.255 & 185.690 & -0.013 \\
\hline & \multirow[t]{4}{*}{ il2 } & A9 & $7.543 e+06$ & $6.102 \mathrm{e}+08$ & 244.686 & 303.342 & 156.920 & 188.508 & 0.047 \\
\hline & & A10 & $1.202 \mathrm{e}+07$ & $6.560 \mathrm{e}+08$ & 251.192 & 305.774 & 156.005 & 184.933 & -0.009 \\
\hline & & $\bar{A} 24$ & $1.105 \mathrm{e}+07$ & $7.020 e+08$ & 250.207 & 298.301 & 157.420 & 185.643 & 0.034 \\
\hline & & A23 & $1.302 \mathrm{e}+07$ & $6.683 e+08$ & 249.343 & 306.203 & 156.469 & 184.862 & 0.015 \\
\hline \multirow[t]{8}{*}{7072} & \multirow[t]{4}{*}{ il1 } & $\overline{\text { A22 }}$ & $5.642 \mathrm{e}+06$ & $4.777 \mathrm{e}+08$ & 281.502 & 262.112 & 160.577 & 185.648 & 0.029 \\
\hline & & A21 & $8.228 \mathrm{e}+07$ & $4.761 \mathrm{e}+08$ & 286.732 & 260.207 & 157.230 & 184.295 & -0.023 \\
\hline & & A2 & $8.639 \mathrm{e}+06$ & $4.157 \mathrm{e}+08$ & 280.448 & 258.338 & 156.920 & 184.623 & 0.048 \\
\hline & & $\overline{\mathrm{A} 4}$ & $1.063 \mathrm{e}+07$ & $4.524 \mathrm{e}+08$ & 287.645 & 259.231 & 154.947 & 183.115 & -0.024 \\
\hline & \multirow[t]{4}{*}{ i12 } & A28 & $6.738 \mathrm{e}+07$ & $4.601 \mathrm{e}+08$ & 254.371 & 296.208 & 159.158 & 185.606 & 0.019 \\
\hline & & A29 & $4.978 \mathrm{e}+06$ & $4.427 e+08$ & 259.120 & 300.104 & 161.769 & 186.055 & -0.003 \\
\hline & & $\bar{A} 31$ & $6.917 \mathrm{e}+06$ & $4.088 \mathrm{e}+08$ & 255.663 & 295.880 & 159.528 & 183.678 & 0.019 \\
\hline & & A30 & $4.368 e+06$ & $4.356 e+08$ & 255.846 & 292.942 & 163.746 & 185.637 & -0.052 \\
\hline \multirow[t]{4}{*}{7130} & \multirow[t]{4}{*}{ il1 } & $\overline{A 99}$ & $1.248 \mathrm{e}+07$ & $5.766 e+08$ & 315.618 & 253.233 & 154.448 & 191.508 & 0.020 \\
\hline & & $\mathrm{A} 10$ & $1.140 \mathrm{e}+07$ & $5.879 e+08$ & 316.443 & 257.476 & 157.409 & 190.006 & -0.001 \\
\hline & & A34 & $8.896 e+06$ & $5.119 e+08$ & 312.565 & 257.819 & 157.020 & 194.261 & 0.031 \\
\hline & & A33 & $9.530 \mathrm{e}+06$ & $5.737 e+08$ & 312.926 & 255.098 & 156.608 & 190.561 & -0.020 \\
\hline \multirow[t]{4}{*}{7209} & \multirow[t]{4}{*}{ il1 } & A29 & $2.625 \mathrm{e}+06$ & $1.558 \mathrm{e}+08$ & 283.047 & 256.653 & 153.212 & 191.656 & 0.009 \\
\hline & & A30 & $3.677 \mathrm{e}+06$ & $1.444 \mathrm{e}+08$ & 286.984 & 258.138 & 152.339 & 188.120 & -0.003 \\
\hline & & A9 & $2.668 \mathrm{e}+06$ & $1.463 e+08$ & 282.241 & 255.786 & 152.708 & 190.008 & 0.022 \\
\hline & & A10 & $2.675 \mathrm{e}+06$ & $1.294 \mathrm{e}+08$ & 288.425 & 252.069 & 151.583 & 191.562 & -0.005 \\
\hline
\end{tabular}

Table 2: Parameter values for the beam shots 


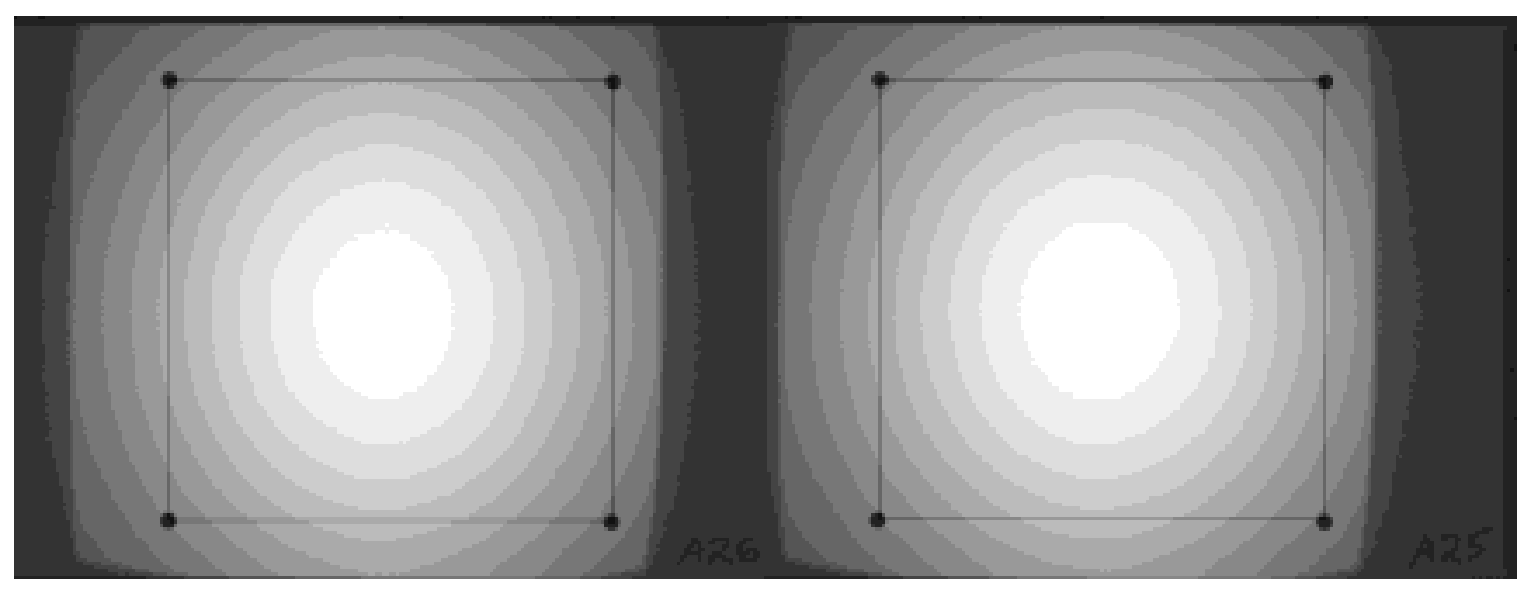

Fig. 4: (Run 7061) Data is "warped"

- Non-stationarity of beam profile. Using the data on plate A4 at run $7000(8 / 2000: 10)$ resulted in $\sigma_{x}=153.406$ and $\sigma_{y}=174.728$, whereas the same plate at run $7072\left(8 / 20\right.$ 06:00) gives $\sigma_{x}=154.947$ and $\sigma_{y}=183.115$. These are changes of $1 \%$ and $4.8 \%$ respectively.

- Unobscured Shots and the Inadequacy of Gaussian Model. To simulate the process of fitting a Gaussian to the blank (unobscured) spots in step wedge shots, we chopped the squares of data (within the holes, pictured in Fig. 3 on page 5) into vertical slices and attempted to fit a Gaussian to those slices using IDL's gauss2dfit. We chopped the data from run 7061, plates A25 and A26 into six strips, and found that the resulting $\sigma_{x}$ values varied (relatively) by $8.0 \%$ and $6.8 \%$ respectively, and the $\sigma_{y}$ values by $4.0 \%$ and $2.8 \%$. This result indicates that our model is not rich enough to capture the RECORDED images. "Recorded" is emphasized, because the record is a combination of beam and plate response. (At more than six slices, the IDL function failed to converge to a solution.)

- Figures 5 and 6 : Fig. 5 (on the next page) shows the last six good beam shots as a function of time. Each frame superimposes one new beam contour. The contour chosen was the $\exp (-.125) *\{\operatorname{Max}\}$ (maximum of Gaussian) level curve. Fig. 6 (on page 10) shows the variation of the $\sigma_{x}^{\prime} s$ 
3 ANALYSIS AND RESULTS

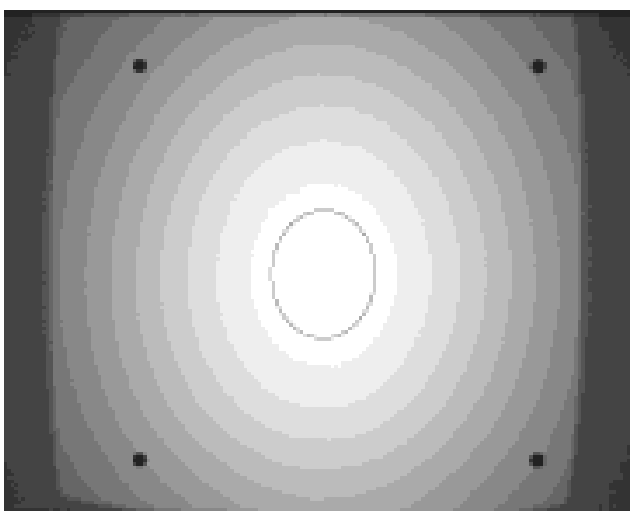

(a) run $7050,8 / 2020: 45$

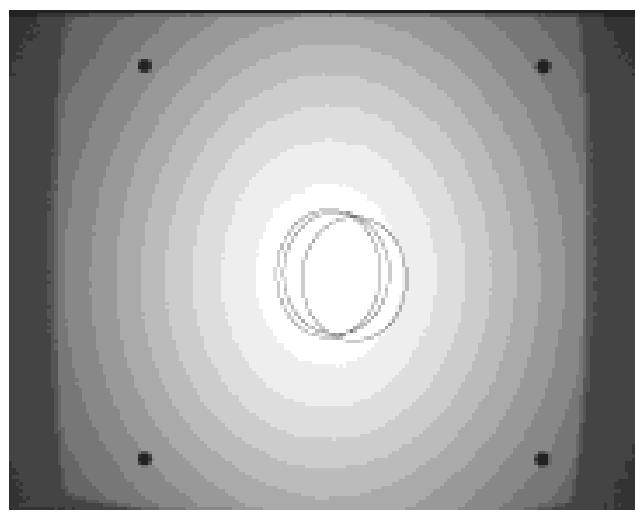

(c) run 7066, 8/21 02:50

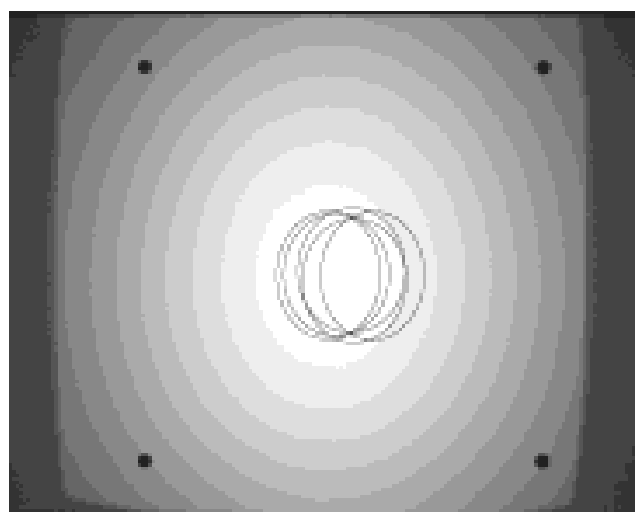

(e) run $7130,8 / 22$ 05:50

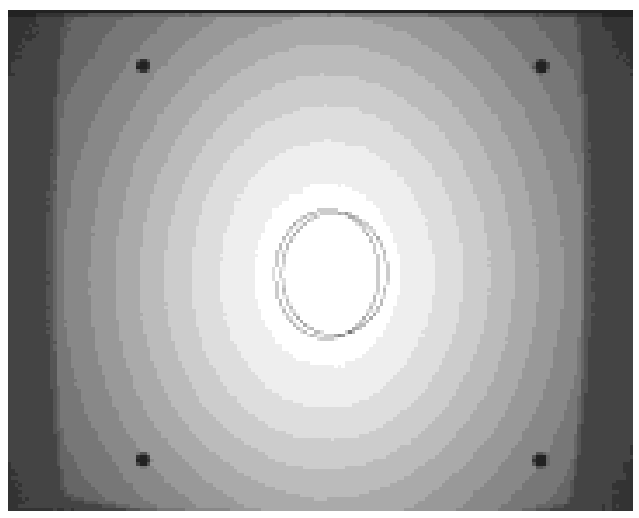

(b) run $7061,8 / 20$ 23:00

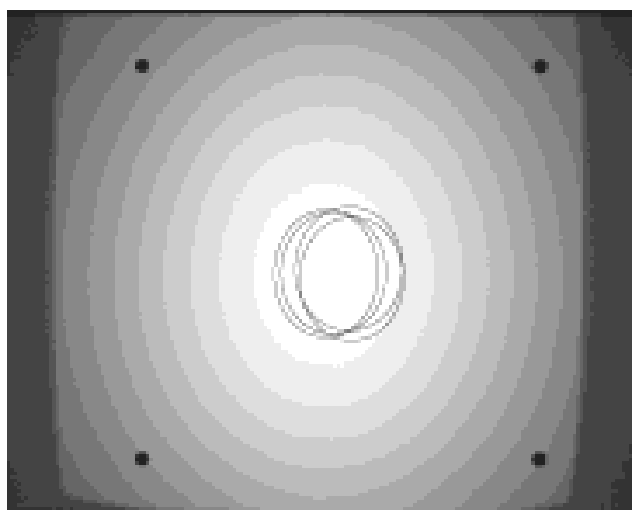

(d) run $7072,8 / 2106: 00$

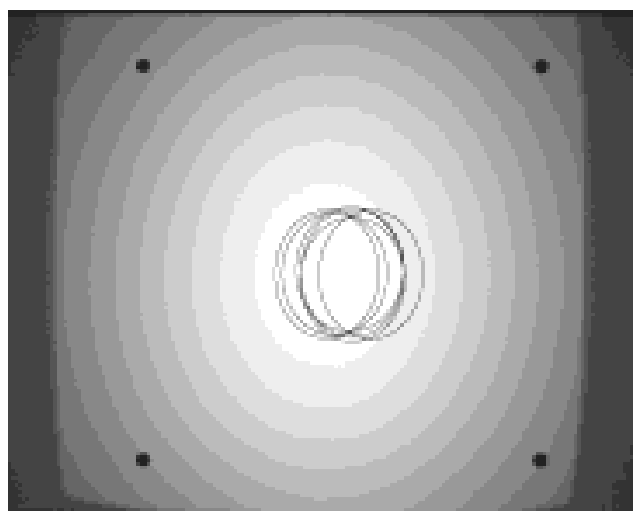

(f) $\operatorname{run} 7209,8 / 23$ 05:00

Fig. 5: Location of beam over time 
and the $\sigma_{y}^{\prime} s$ as a function of time. This plot includes all the data from measurements made at image plane 1 .

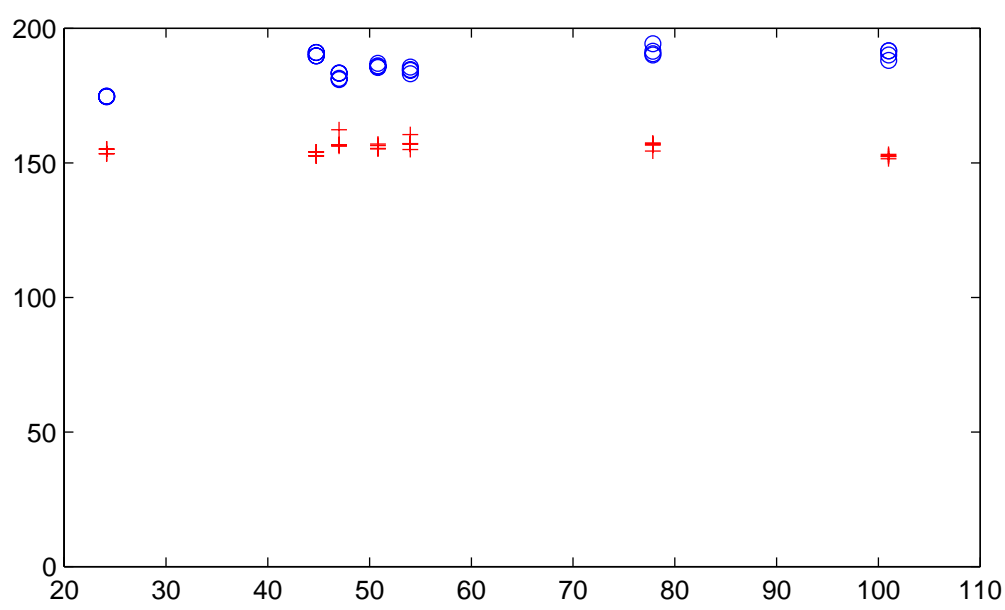

Fig. 6: Variation of $\sigma_{x}$ (+'s) and $\sigma_{y}$ (circles)

Remark: One can expect the values to change slightly due to measurement noise, but the effect of noise is very small. For example, the $\sigma_{y}$ numbers differ (relatively) by about $4.5 \%$, whereas if we add $1 \%$ random noise to data and fit a Gaussian, the $\sigma$-values change (relatively) by about $0.1 \%$.

Remark: In order to overlay the measurements from multiple plates (taken at the same time), there is a substantial amount of preprocessing required. This requirement is caused by the variability between the plates themselves (for example, the difference between plates A21 and A22 as described on page 6), and by the varying dimensions within the TIFF files as illustrated in Fig. 4.

\section{Next Steps}

The results in the previous section reveal multiple sources of uncertainty that must be characterized or eliminated in order to obtain tightly constrained final numbers. The next steps or tasks are clearly indicated by the above results. Ultimately these tasks, which are outlined below, will generate 
recommendations and new analysis algorithms. It may be the case that this work will result in recommendations of radical changes to the experimental procedure. More optimistically, our recommendations will entail only low-cost experimental modifications and innovative analysis procedures.

We now turn to a list of tasks to be completed next.

- Analysis Tasks:

- A rigorous sensitivity analysis of the step wedge reconstruction will be carried out. Observed variations - for example, beam profiles variations - will then be propagated forward to see the effect of those variations on the final numbers of interest.

- A careful study of the flatness or non-flatness of the plate-response will be done next. This work will be done by studying the change of response ratios of pairs of plates from shot to shot (beam and non-beam alike). There will also be carefully designed beam profile experiments at LANSCE (more on this below).

- Once the variability is in hand and we have rigorous error bars on our estimates of the beam profile and plate sensitivities, we can ask what effect these error bars have on the reconstructed densities. This effect can be calculated by propagating the error bars forward through a magnification factor determined by the inversion method.

- The response variations will be studied to see if they correlate with overall plate record shape changes. (A higher response due to buckling away from drum should lead to a shorter image in the TIFF file.)

- A valid model for the beam must be identified because masked (partly obscured) shots depend on the quality of this model for the purposes of beam profile estimation from the intensities at unobscured portions of the radiograph.

- Experimental tasks:

- We will design and carry out a sequence of experiments at LANSCE using a combination of cameras and plates in an attempt to completely characterize the plate responses. The studies goal is the 
5 SUMMARY AND OUTLOOK

successful characterization of the plate responses for each plate. Success means that whatever uncertainties remain will be parameterized in such a way that they can be estimated accurately from experimental shots. It appears we will have to include the design of new procedures to eliminate some of the variability that enters now in the scanning of the plates.

- Fuji will be contacted about the fabrication process of the plates to see if the phosphor concentration is tightly controlled and the distribution is always even across each plate.

- Beam data from Ed Hartouni at Lawrence Livermore will be obtained. We will look into the possibility of doing an experiment to characterize the transfer function that converts a beam spot profile into a post-diffuser profile.

- The entire process, starting with the plate exposure and ending with the drum scanning and plate erasure will be reviewed in an attempt to pin down the anomalous variation in plate response (total response).

\section{Summary and outlook}

In this preliminary study we found significant variation in the measured beam profiles collected at BNL. Not only was there a drift in the beam centers, there were also significant variations in the beam shape $\left(\sigma_{x}\right.$ and $\left.\sigma_{y}\right)$. What must be kept in mind is that the variations observed so far are a combination of beam variation, plate response variations and scanning inaccuracies. These effects will only be untangled by the more extensive in-depth study we have suggested and are in fact carrying out.

The observed variations and the known coupling of factors contributing to those variations clearly indicates the need for further in-depth study. We give specific guidelines as to what steps should be taken. The first step is a detailed, rigorous sensitivity analysis of the step wedge reconstruction process. This step will permit us to propagate observed variations and uncertainties forward to obtain error bars on the final numbers. In turn, this result will permit us to find improvements in parameterizations, experimental procedure, etc., that will result in smaller error bars. 


\section{SUMMARY AND OUTLOOK}

An accurate parameterized beam model, a precise understanding of the relationship between the pre- and post-diffuser beam profiles, and a record of the post-diffuser beam profile each potentially permit the beam profile to be subtracted from the recorded object radiographs. Each of these will be pursued since it is not clear which will lead to the desired result.

Finally, almost as a side effect of these studies and experiments, we will be in a position to generate definitive reconstructions of the experimental objects studied at BNL in August of 1999. These reconstructions will include tight, rigorous error bars. 
REFERENCES

\section{References}

[1] Alexander Saunders. A study of plate response and variability. P-25, Los Alamos National Laboratory.

[2] BNL E933 unclassified log books. Available online at https://pcprad9. lanl.gov/RunLogs/Runs 1999/LogBooks/.

[3] BNL E933 unclassified data. Available online at https://pcprad9.lanl.gov/RunLogs/praddataags1999/. 